\title{
Effects of Polymer Gel Electrolyte on Photoelectric Properties and Driving Characteristics of Prussian-Blue-Based Electrochromic Cells in Automatic Driving Circuit
}

\author{
Syo Miyagi, Takuya Kusanagi, and Shuichi Sato* \\ Department of Electronic Engineering, Tokyo Denki University, \\ 5 Senju-Asahi-cho, Adachi-ku, Tokyo 120-8551, Japan \\ *s.sato@mail.dendai.ac.jp
}

\begin{abstract}
The effects of polymer gel electrolyte on the photoelectric and driving characteristics of Prussian blue (PB)-based electrochromic (EC) cells were systematically investigated under different preparation conditions. The EC cells were switched from the coloring to decoloring mode by applying $\pm 1.5 \mathrm{~V}$. Subsequently, we prepared an automatic driving circuit wherein the signal of the voltage was alternately applied. The thickness of the PB layer significantly depended on the switching speed as opposed to the electrolyte concentration and the polymer gel electrolyte. In particular, the speed of coloring exceeds that of decoloring due to the electron exchange in the chemical reaction. The speed varied from approximately 5 to 10 times based on the type of the electrolyte.
\end{abstract}

Keywords: Electrochromic, Prussian blue, Stability, Gel electrolyte, Automatic driving circuit

\section{Introduction}

Optical devices including televisions and cellphones play an important role in broadcasting systems and information technology industries. Various technologies, such as liquid crystal [1,2], plasma [3], inorganic electroluminescence [4,5], and organic electroluminescence [6,7], are developed for the fabrication of optical displays. These displays are classified as transmissive displays.

With respect to a transmissive display, a light receiving (reflective) display has recently attracted significant attention. An important example of this type of display is the "electronic paper". It is possible to see images more naturally even in bright places without the effect of the viewing angle. In addition, it is possible to continue displaying the image even after the voltage application is released (memory function), and it is driven with low energy. Therefore, several studies and developments including that of light control glass focus on the same [8].

In this study, we examine electrochromism that is increasingly used for light control glass.
Specifically, electrochromism is a reaction in which color is changed by voltage application, and subsequently it keeps the change without maintaining applied voltage. We focus on Prussian blue (PB) that corresponds to a low coat material wherein it is easy to prepare the film and it can be driven with low voltage [9].

A low voltage drivable electrochromic (EC) cell as reported in extant studies [10] is shown in Fig. 1. Specifically, PB and nickel hexacyanoferrate (NiPBA) are coated on the transparent electrode, and an electrolyte layer containing $\mathrm{K}^{+}$is formed between the two transparent electrodes. The crystal structures of PB and Ni-PBA fabricated via structure visualization software VESTA are shown in Fig. 2 [11,12]. The crystal structure of PB is cubic as shown in Fig. 2(a) and is crosslinked with $\mathrm{Fe}^{2+}-\mathrm{CN}-\mathrm{Fe}^{3+}$. Specifically, $\mathrm{PB}$ exhibits a $\mathrm{Fe}(\mathrm{II})(\mathrm{CN})_{6}$ missing in the unit lattice, and an insoluble PB is prepared. The crystal easily captures $\mathrm{K}^{+}$due to the neutral charge. Therefore, an electrolyte containing $\mathrm{K}^{+}$is adopted as the electrolyte layer of the EC cell.

As shown in Fig. 2(b), the crystal structure of Ni- 


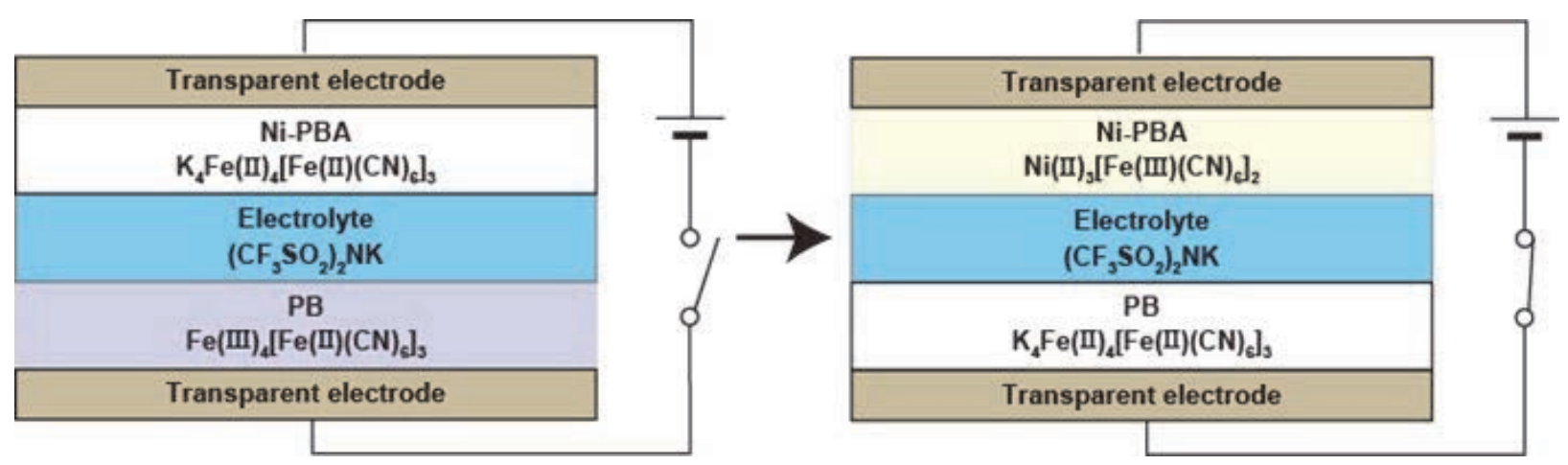

Fig. 1. Cell structures and chemical reactions of the PB-based EC cell.

PBA is a structure in which $\mathrm{Fe}^{3+}$ is replaced by $\mathrm{Ni}^{2+}$. The reactions at the time of application of DC voltage are summarized below [13].

Cathode :

$\begin{aligned} \mathrm{Fe}(\mathrm{III})_{4}\left[\mathrm{Fe}(\mathrm{III})(\mathrm{CN})_{6}\right]_{3}(\text { blue })+4 \mathrm{e}^{-}+4 \mathrm{~K}^{+} & \\ & \rightarrow \mathrm{K}_{4} \mathrm{Fe}(\mathrm{II})_{4}\left[\mathrm{Fe}(\mathrm{II})(\mathrm{CN})_{6}\right]_{3} \text { (colorless) }\end{aligned}$

Anode :

$\mathrm{K}_{2} \mathrm{Ni}(\mathrm{II})_{3}\left[\mathrm{Fe}(\mathrm{II})(\mathrm{CN})_{6}\right]_{2}$ (colorless)

$\rightarrow \mathrm{Ni}(\mathrm{II})_{3}\left[\mathrm{Fe}(\mathrm{III})(\mathrm{CN})_{6}\right]_{2}$ (yellow) $+2 \mathrm{e}^{-}+2 \mathrm{~K}^{+}$

When $\mathrm{Fe}^{2+}$ turns into $\mathrm{Fe}^{3+}$, the Ni-PBA film emits electrons and exhibits a yellow color. Conversely, electrons and ions enter the PB film, and the film is reduced and decolored. Given that the Ni-PBA film is very light in color, it appears transparent after the DC voltage application.
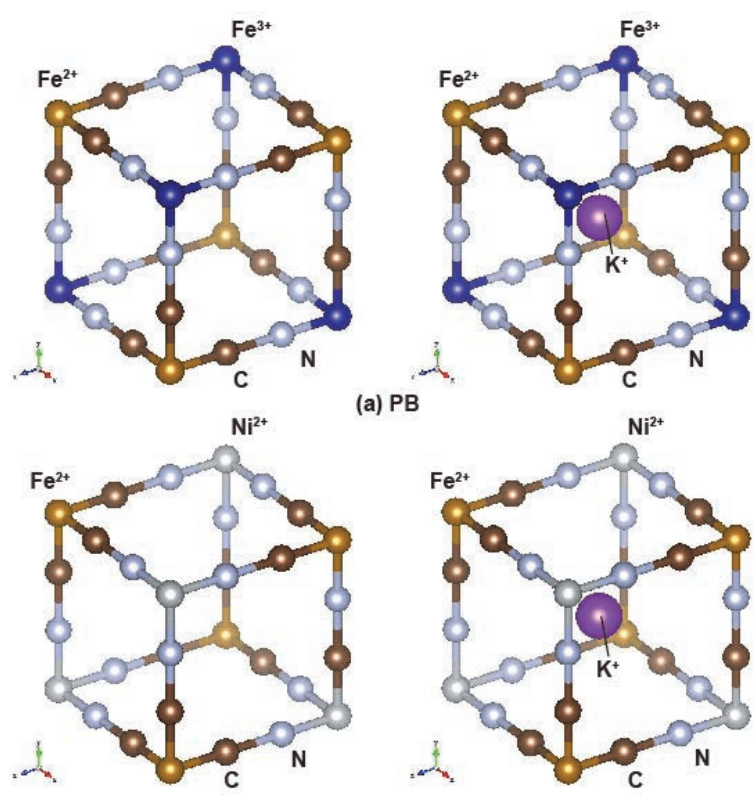

(b) Ni-PBA

Fig. 2. Crystalline structures of (a) PB and (b) NiPBA.
Therefore, in the study, we fabricated PB-based EC cells and systematically examined the effect of film forming conditions and electrolytic solution concentration on electric characteristics and driving characteristics. A normal electrolyte was prepared, and a polymer gel electrolyte was prepared to further reduce the risk of leakage. We systematically investigated the effect of each on the characteristics. This is because there is a paucity of extant studies that systematically compare the basic characteristics of EC cells. Additionally, a small driving circuit that is easily manufactured at low cost was also fabricated for the development of the EC device in the study. The development of EC materials and research on driving circuits is conducted independently. The proposed improvements in the driving circuit are expected to significantly contribute to research on EC devices.

\section{Experimental}

2.1. Sample preparation

For this study, PB was purchased from Kanto Chemical Co., Tokyo, Japan, and prepared by mixing $9 \mathrm{wt} \%$ of FeHCF nanoparticles in water. Additionally, Ni-PBA was purchased from Kanto Chemical Co., Tokyo, Japan, and prepared by mixing $8 \mathrm{wt} \%$ of NiHCF nanoparticles into the water. Potassium bis(trifluoromethanesulfonyl) imide (Kanto Chemical Co., Tokyo, Japan) was used as the electrolyte. Sedimentation velocity as a measure of the viscosity of PB and Ni-PBA was measured with a dispersibility evaluation particle size distribution analyzer LUMiSizer (LUM Japan Co., Ltd., Tokyo, Japan), and is shown in Fig. 3. The movement of the position of the interface inside the sample container is shown. The sedimentation velocity of Ni-PBA (Fig. 3(b)) exceeded that of PB (Fig. 3(a)). Given that PB exhibits large particles that cannot be measured by the devices using light transmission, it was filtered using a membrane filter with a pore diameter of $5.0 \mu \mathrm{m}$ and used in the 

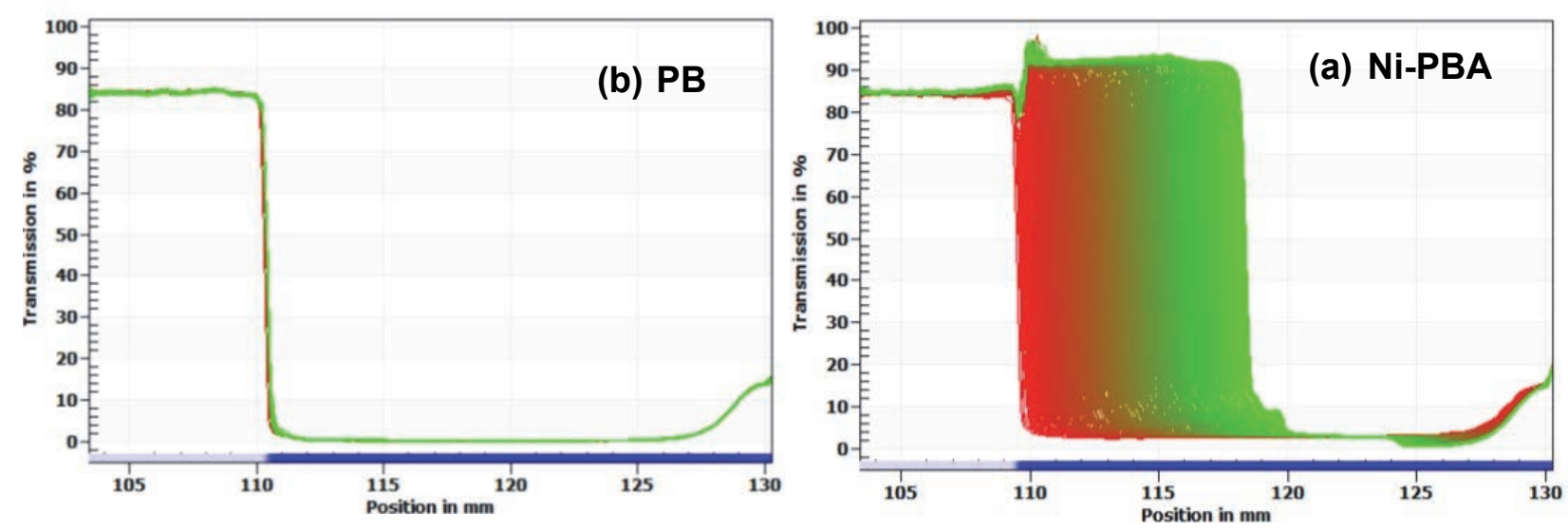

Fig. 3. Sedimentation velocity of PB (a) and Ni-PBA (b) inks.

experiment.

Propylene carbonate (Kanto Chemical Co. Inc., Tokyo, Japan) was used as the solvent to adjust the electrolyte concentration. Polymethyl methacrylate (PMMA) (Kanto Chemical Co. Inc., Tokyo, Japan) was used for gelling the electrolyte.

Finally, indium tin oxide (ITO) glass (E.H.C. Co., Tokyo Japan.) was used as the transparent electrode. It exhibited a resistance of $5 \Omega$ /square with a thickness of $0.7 \mathrm{~mm}$ and area of $30 \times 40 \mathrm{~mm}$.

While preparing the EC cell in Fig. 1, eight different production conditions are examined. The ITO substrate was cleaned with alcohol and subjected to hydrophilic treatment by plasma for approximately $1 \mathrm{~min}$. Subsequently, PB films were formed at $1500 \mathrm{rpm}$ or $7500 \mathrm{rpm}$ for $20 \mathrm{~s}$ via the spin coating method using MS-A 100 spin coater (Mikasa Co., Ltd., Tokyo Japan). All Ni-PBA films were formed at $1500 \mathrm{rpm}$ for $20 \mathrm{~s}$.
Potassium bis(trifluoromethanesulfonyl)imide $\left(\left(\mathrm{CF}_{3} \mathrm{SO}_{2}\right)_{2} \mathrm{NK}\right)$ exhibiting a concentration of 0.1 $\mathrm{mol} / \mathrm{L}$ or $0.5 \mathrm{~mol} / \mathrm{L}$ was dissolved in propylene carbonate. The ITO substrates with the PB film and with the Ni-PBA film were adhered at intervals of $100 \mu \mathrm{m}$. Additionally, an electrolyte layer was formed. The gel electrolyte was prepared by mixing $30 \mathrm{wt} \%$ of PMMA in the electrolyte prepared earlier at $95^{\circ} \mathrm{C}$ for $24 \mathrm{~h}$.

The thickness of the Ni-PBA film of all the EC cells was maintained as constant. Additionally, EC cells with the thick PB film and the thin PB film were prepared. Furthermore, EC cells with high electrolyte concentration and with low electrolyte concentration were prepared. Moreover, EC cells using electrolyte and using a gel electrolyte were prepared. A total of eight types of EC cells were prepared. The preparation conditions are summarized in Table 1.

Table 1. Preparation conditions of the EC cell.

\begin{tabular}{cccc}
\hline Cell No. & $\begin{array}{c}\text { Spin-coating speed } \\
(\mathrm{rpm})\end{array}$ & Electrolytes & $\begin{array}{c}\text { Electrolyte concentration } \\
(\mathrm{mol} / \mathrm{L})\end{array}$ \\
\hline 1 & 1500 & Electrolyte & 0.1 \\
2 & 1500 & Electrolyte & 0.5 \\
3 & 7500 & Electrolyte & 0.1 \\
4 & 7500 & Electrolyte & 0.5 \\
\hline 5 & 1500 & Gel-Electrolyte & 0.1 \\
6 & 1500 & Gel-Electrolyte & 0.5 \\
7 & 7500 & Gel-Electrolyte & 0.1 \\
8 & 7500 & Gel-Electrolyte & 0.5 \\
\hline
\end{tabular}


Specifically, in EC cell No.1, the PB film was formed at $1500 \mathrm{rpm}$. The electrolyte concentration corresponded to $0.1 \mathrm{~mol} / \mathrm{L}$. In EC cell No.2, the PB film was formed at $1500 \mathrm{rpm}$. The electrolyte concentration corresponded to $0.5 \mathrm{~mol} / \mathrm{L}$. In EC cell No.3, the PB film was formed at $7500 \mathrm{rpm}$. The electrolyte concentration corresponded to 0.1 $\mathrm{mol} / \mathrm{L}$. In EC cell No.4, the PB film was formed at $7500 \mathrm{rpm}$. The electrolyte concentration corresponded to $0.5 \mathrm{~mol} / \mathrm{L}$. In EC cell No.5, the PB film was formed at $1500 \mathrm{rpm}$. The gel electrolyte concentration corresponded to $0.1 \mathrm{~mol} / \mathrm{L}$. In EC cell No.6, the PB film was formed at 1500 rpm. The gel electrolyte concentration corresponded to $0.5 \mathrm{~mol} / \mathrm{L}$. In EC cell No.7, the PB film was formed at $7500 \mathrm{rpm}$. The gel electrolyte concentration corresponded to $0.1 \mathrm{~mol} / \mathrm{L}$. In EC cell No.8, the PB film was formed at 7500 rpm. The gel electrolyte concentration corresponded to $0.5 \mathrm{~mol} / \mathrm{L}$.

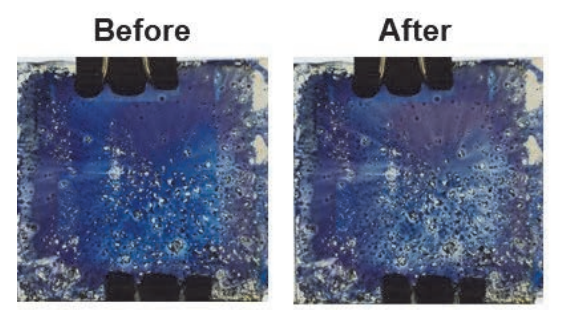

No. 1
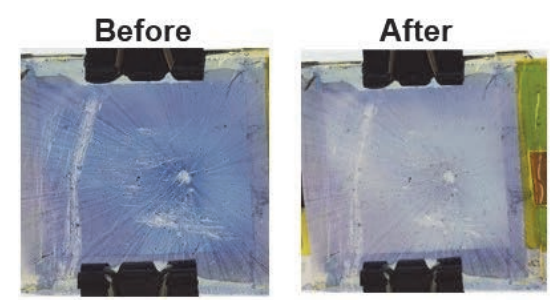

No. 3

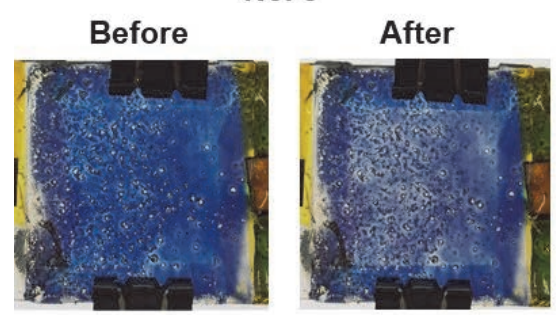

No. 5

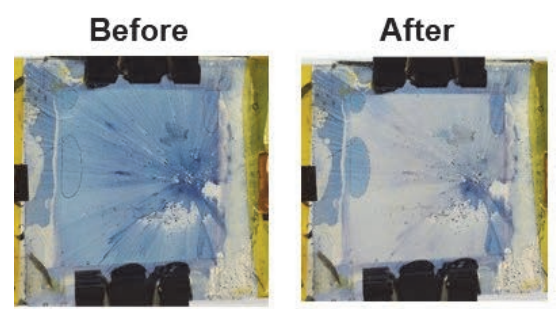

No. 7
2.2. Electrical properties of electrochromic cells

The impedance at each frequency of the eight types of EC cells was measured via a chemical impedance analyzer (IM 3590, Hioki E. E. Co., Nagano, Japan). Measurement was performed in the range of $0.1 \mathrm{~Hz}$ to $200 \mathrm{kHz}$. The applied voltage was set as $1 \mathrm{~V}$ to avoid deterioration of the EC cell under measurement. Cyclic voltammetry was performed with ALS/DY 2325 bipotentiostat (BAS Inc., Tokyo, Japan). It was swept linearly from $-1.5 \mathrm{~V}$ to $+1.5 \mathrm{~V}$ and repeated twice to confirm the reproducibility of the experimental results. The measurement of driving characteristics was performed by measuring the voltage with an oscilloscope (TDS 2022 B, Tektronix, Inc., Beaverton, Oregon, USA).

\section{Results and discussion}

3.1. Electrical properties of electrochromic cells

Figure 4 displays the photographs depicting the

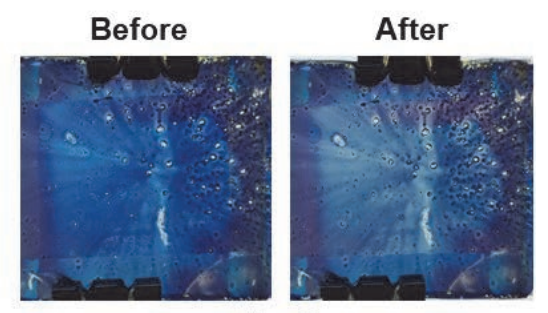

No. 2

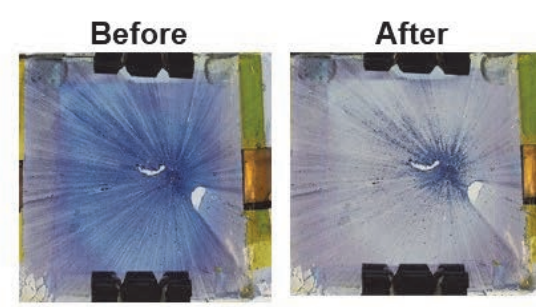

No. 4

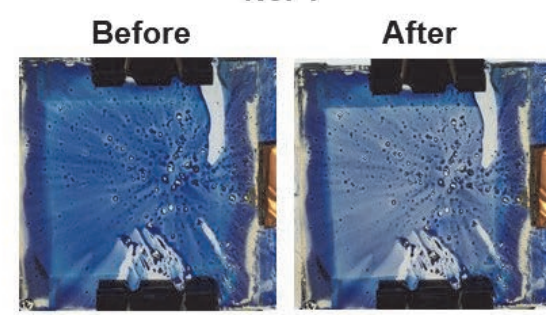

No. 6

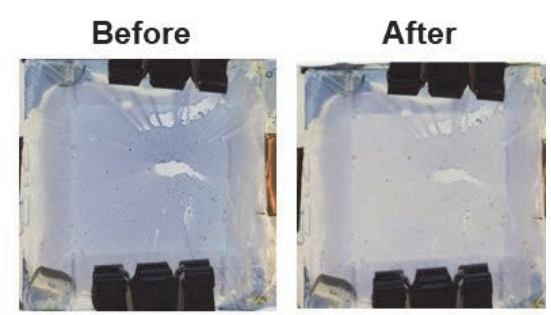

No. 8

Fig. 4. Switching images of EC cells with the application of DC voltage of $\pm 1.5 \mathrm{~V}$. 
changes in appearance as the EC cells are driven at $\pm 1.5 \mathrm{~V}$. With respect to the applied voltage of 1.5 $\mathrm{V}$, decoloring at the cathode due to injection of electrons into the PB film was observed in all the EC cells. With respect to the applied voltage of $1.5 \mathrm{~V}$, coloring at the cathode due to the oxidation of the PB film was observed in all the EC cells. The decoloring was unclear in EC cells Nos. 1 and 5. However, in EC cells Nos. 2 and 6 with a high electrolyte concentration, an evident change (switching from coloring and decoloring state) was observed. In EC cells Nos. 3, 4, 7, and 8 with a thin PB film, decoloring was evident in all cases. EC cells Nos. 1 and 5, Nos. 2 and 6, Nos. 3 and 7, Nos. 4 and 8 display approximately the same degree of sharpness of decoloring. Gelling did not affect the appearance.

With respect to the frequency dependence of the impedance $Z$ and phase $\theta$ of the EC cell, the result of the EC cell with the electrolyte is shown in Fig. 5 (a), and the result of the EC cell with the gel electrolyte is shown in Fig. 5(b). The impedance $Z$ of the EC cell corresponded to $132-195 \Omega$ in the low frequency region of EC cells Nos. 1-4. The order of $Z$ corresponded to EC cells No. $1>$ No. $2>$ No. $3>$ No. 4 . Specifically, $Z$ decreased when the thickness of the PB layer reduced and the electrolyte concentration increased. With respect to Nos. 1 and 2, where the PB film was thick, it slowly decreased once around $10 \mathrm{~Hz}$ and subsequently decreased with increases in the frequency. In the high frequency region, the value converged to 17.2 $\Omega, 15.4 \Omega, 15.7 \Omega$, and $13.3 \Omega$ for EC cell Nos. 1,2 , 3 , and 4 , respectively. In EC cells, using the gel electrolyte, $Z$ approximately corresponded to 105 $185 \Omega$ in the low frequency region. Additionally, the value of $Z$ converged to $27.2 \Omega, 18.5 \Omega, 24.9 \Omega$, and $15.5 \Omega$ for EC cell Nos. 5, 6, 7, and 8, respectively. Their trajectories were almost identical.

The phase $\theta$ due to the resistance and capacitance components of the EC cell was $-56^{\circ}--63^{\circ}$ in the low frequency region of EC cell Nos. 1-4, and the capacitance component was strong. However, in the high frequency region, it converged to $0^{\circ}$ and shifted to the resistance component. For EC cell Nos. 1 and 2, the shift was moderate at approximately $10 \mathrm{~Hz}$. Conversely, EC cell Nos. 5-8 using the gel electrolyte, a shift from the capacitance component to the resistance component was observed. Their trajectories were almost identical, and the component as the element (equivalent circuit) was stable without depending on the preparation conditions.

Based on the impedance characteristics, an
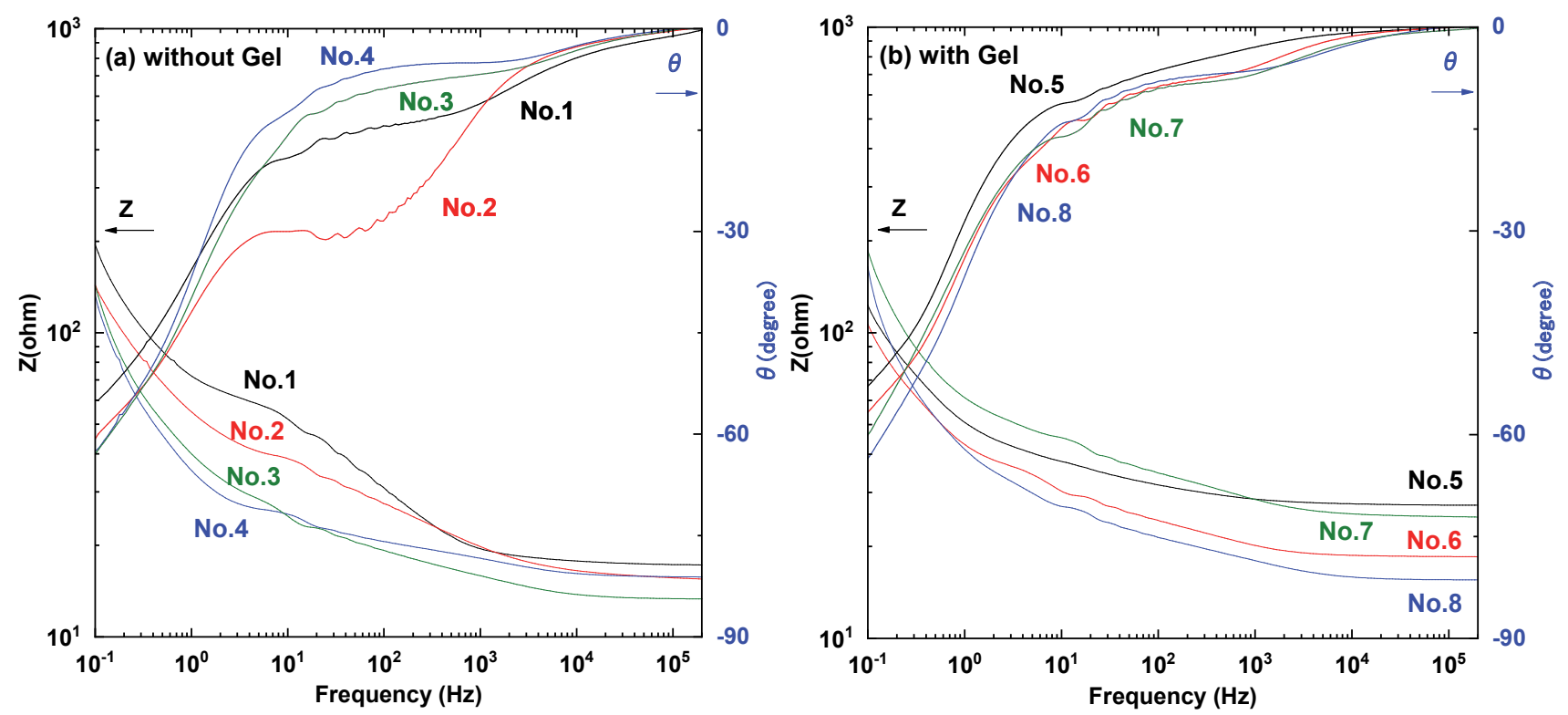

Fig. 5. Impedance and phase of the EC cells (a) without polymer gel electrolyte and (b) with the gel. 


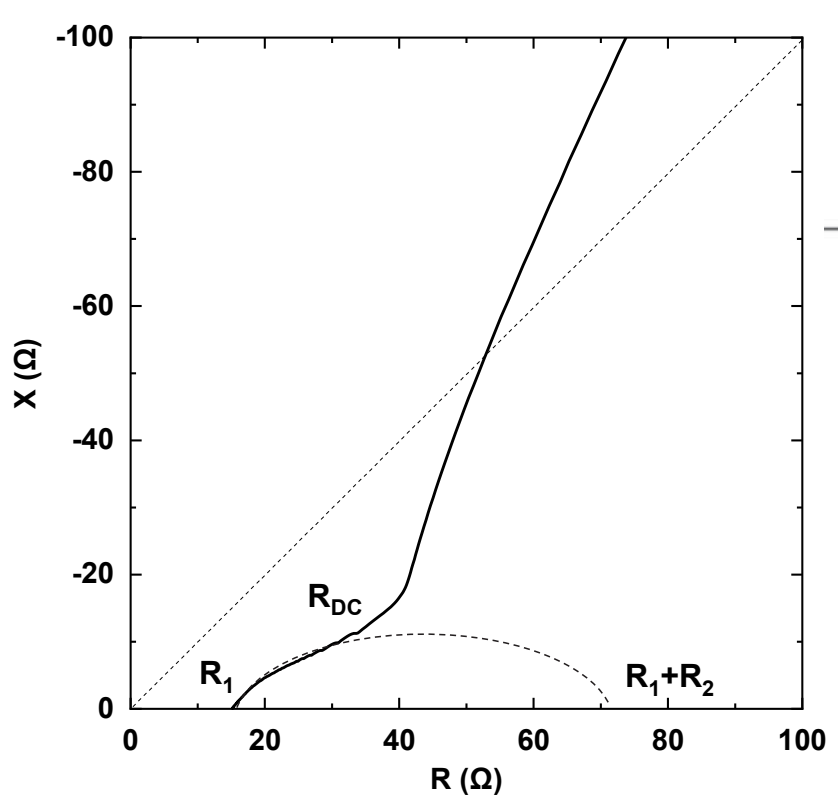

Fig. 6. Cole-Cole plot of the EC cell.

equivalent circuit analysis was performed. The equivalent circuit was created by comparing the impedance characteristics with the model.

Figure 6 shows the Cole-Cole plot, which is a vector trajectory that represents the real part of resistance $R \Omega$ on the $\mathrm{X}$ axis and the imaginary part of resistance $X \Omega$ on the $\mathrm{Y}$ axis. Sample No. 1 is shown as an example. A semicircle was drawn mid-way, and the capacitance component became strong at a certain point such that it corresponded to a locus showing a straight line. Thus, in the case of the EC cell used in this study, the equivalent circuit corresponded to a circuit as shown in Fig. 7 and was in agreement with extant studies [14]. A resistor $R_{l}$ (two in Fig. 7) is connected in series to a parallel circuit of the electric double layer capacitance $C_{p}$, resistor $R_{2}$, and Warburg impedance $Z_{w}$ (diffusion impedance assuming linear semiinfinite diffusion).

The impedance $Z(\omega)$ of the entire equivalent circuit shown in Fig. 7 is expressed in Eq. (1) where $\omega$ denotes the angular frequency as follows:

$$
Z(\omega)=R_{1}+\frac{1}{j \omega C_{p}+\frac{1}{R_{2}+Z_{W}}}
$$

In the high frequency region, $Z(\omega)$ decreases and $R_{2} \gg Z_{w}$, and Eq. (1) is transformed into Eq. (2) as follows:
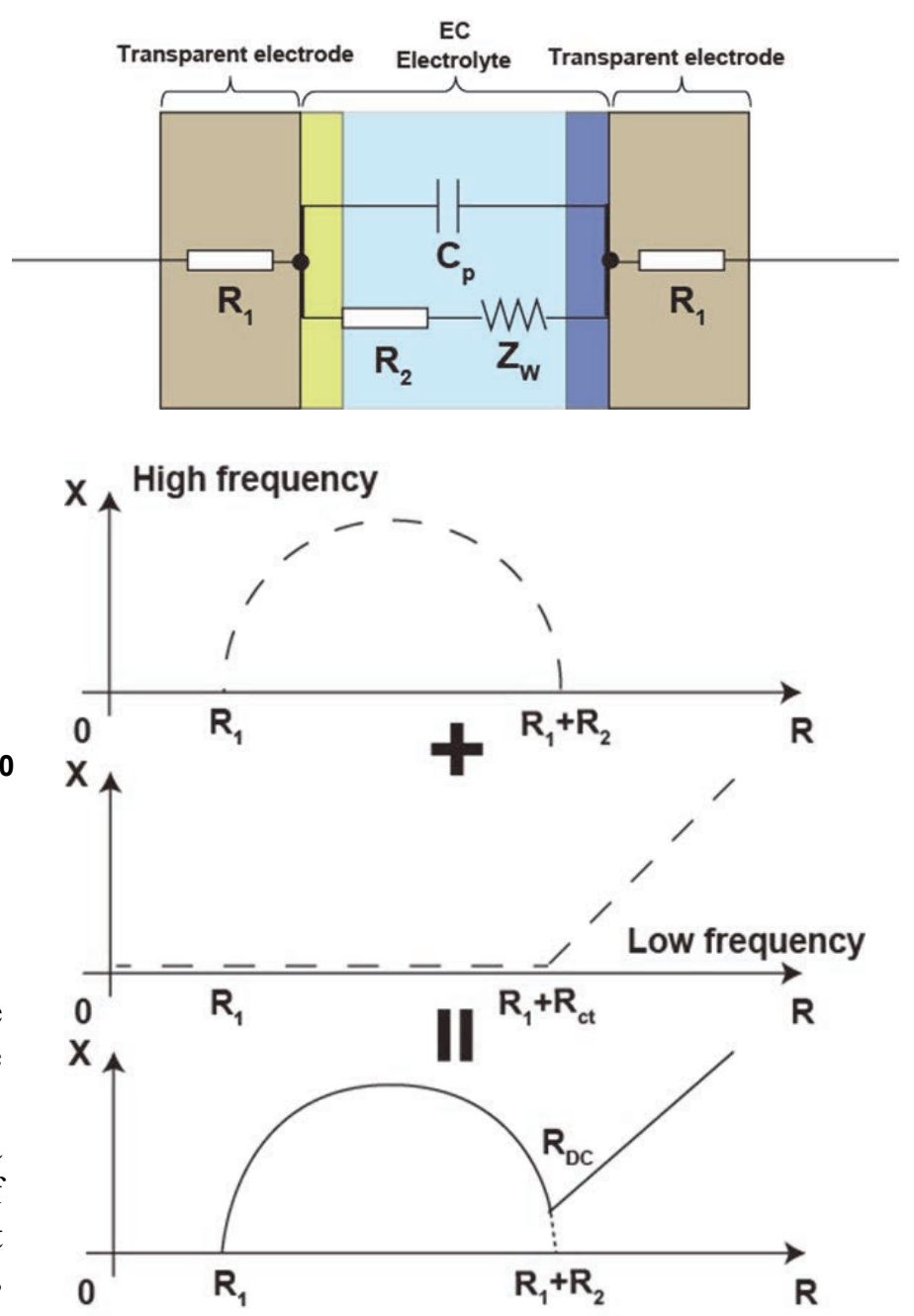

Fig. 7. Equivalent circuit and vector diagram of the EC cells.

$$
Z(\omega) \approx R_{1}+\frac{1}{j \omega C_{p}+\frac{1}{R_{2}}}
$$

Based on the equation, only a semicircle with diameter ranging from $R_{1}$ to $R_{1}+R_{2}$ is drawn as shown in Fig. 7. Conversely, the electrolyte significantly depends on the $Z(\omega)$. Therefore, $1 /\left(R_{2}+Z_{W}\right)$ significantly exceeds $\omega C_{p}$, and Eq. (1) is transformed to Eq. (3) as follows:

$$
Z(\omega) \approx R_{1}+R_{2}+Z_{w}
$$

where is re-expressed as Eq. (4) on the basis of parameter of charge transfer diffusion $\sigma$ and charge transfer resistance $R_{C T}[15]$ as follows:

$$
R_{2}=R_{C T}+\sigma \omega^{-\frac{1}{2}} \quad Z_{w}=\sigma \omega^{-\frac{1}{2}}(1-j)
$$


Thus, Eq. (3) is transformed to Eq. (5) as follows:

$$
\begin{aligned}
Z(\omega) \approx R_{1}+R_{C T}+2 \sigma \omega^{-\frac{1}{2}}-j \sigma \omega^{-\frac{1}{2}} \\
=R_{1}+R_{C T}+\sigma \omega^{-\frac{1}{2}}(2-j)
\end{aligned}
$$

The equation denotes a straight line wherein the inclination is $1 / 2$, and the line originates at $R_{I}+R_{C T}$ on the $\mathrm{X}$ axis. Thus, the figure corresponds to a combination of a semicircle (high frequency region) and straight line (low frequency region). Therefore, the figure shown in Fig. 6 that combines a semicircle and straight line is observed. The semicircle started from $R_{l}$. The cross point between the straight line and semicircle was observed. The value of real part of resistance corresponded to $R_{D C}$ in the cross point. Finally, another intersection point with the $\mathrm{X}$ axis was observed at $R_{1}+R_{2}$. The parameters, $R_{1}, R_{2}$, and $R_{D C}$ are extremely important for the analysis of the EC cells.

Based on the aforementioned equations, the parameters corresponding to $R_{1}, R_{2}$, and $R_{D C}$ of the model in Fig. 7 were calculated. The parameters for EC cell Nos. 1-8 are summarized in Table 2. Specifically, $R_{1}$ exhibited values of $15.7-27.2 \Omega$ in EC cell Nos. 1, 3, 5, and 7, and 13.3-18.5 $\Omega$ in EC cell Nos. 2, 4, 6, and 8. If the film thickness and gelling corresponded to the same condition, then the EC cell with the high electrolyte concentration exhibited a lower value. The value is presumed as due to the resistance of the transparent electrode itself and the surface condition. Thus, $R_{l}$ exhibited values of 15.4-27.2 $\Omega$ in EC cell Nos. 1, 2, 5 and 6 and 13.3-24.9 $\Omega$ in EC cell Nos. 3, 4, 7 and 8. If the electrolyte concentration and gelling corresponded to the same condition, the EC cell with the thin PB film exhibited a lower value. Furthermore, $R_{l}$ exhibited values of 13.3-17.2 $\Omega$ in EC cell Nos. 1-4, and 15.5-27.2 $\Omega$ in EC cell Nos. 5-8. If the film thickness and electrolyte concentration corresponded to the same condition, then the EC cell prepared from the electrolyte exhibited a lower value. Moreover, $R_{2}$ exhibited values of 21.8-47.4 $\Omega$ in EC cell Nos. 1, 3, 5 and 7, and 24.2-53.6 $\Omega$ in EC cell Nos. 2, 4, 6 and 8 . Generally, EC cells with low electrolyte concentration exhibited lower values. This is presumed as due to ionic resistance including interfacial resistance and charge transfer resistance caused by the electrolyte, the PB film, and the NiPBA film.

Finally, we considered $R_{D C}$ based on the results for $R_{1}$ and $R_{2}$. Ideal semicircles and straight lines are not obtained due to the surface roughness, film thickness distribution, and bubbles at the interface. Therefore, with respect to $R_{D C}$, the boundary points between the linear part and arc part are summarized in Table 2. Additionally, $R_{D C}$ was established by balancing $R_{1}$ and $R_{2} ;\left(R_{1}+R_{2}\right)$. In EC cell Nos. $1-3$, the boundary point was on $R_{2}$ side while the $R_{I}$ side was confirmed in EC cell Nos. 4-8.

Table 2. Equivalent circuit parameters of the EC cells.

\begin{tabular}{ccccc}
\hline Cell No. & $R_{1}$ & $R_{1}+R_{2}$ & $R_{2}$ & $R_{D C}$ \\
$(\Omega)$ & $(\Omega)$ & $(\Omega)$ & $(\Omega)$ \\
\hline 1 & 17.2 & 61.4 & 44.2 & 33.3 \\
2 & 15.4 & 69.0 & 53.6 & 43.6 \\
3 & 15.7 & 37.5 & 21.8 & 19.6 \\
4 & 13.3 & 37.9 & 24.6 & 17.3 \\
\hline 5 & 27.2 & 49.4 & 22.2 & 36.8 \\
6 & 18.5 & 43.9 & 25.4 & 21.5 \\
7 & 24.9 & 72.3 & 47.4 & 33.6 \\
8 & 15.5 & 39.7 & 24.2 & 19.6 \\
\hline
\end{tabular}

3.2. Reversible reaction properties of electrochromic cells

Chemical reaction rate and electron transfer rate of EC cells were systematically investigated via cyclic voltammetry. The cyclic voltammograms of the EC cells using the electrolyte are shown in Fig. 8(a), and the cyclic voltammograms of the EC cells using the gel electrolyte are shown in Fig. 8(b). The results confirmed two stages of electron giving and receiving at approximately $-1.5 \mathrm{~V}$ and approximately $+0.2 \mathrm{~V}$ in all the EC cells. At approximately $+0.2 \mathrm{~V}$, both oxidation peak potential and reduction peak potential were confirmed. The results indicated that the vicinity of $+0.2 \mathrm{~V}$ corresponded to the potential at which electrochromism occurred. This was similar to the trend observed in extant studies [16-18].

Although the peak of EC cell Nos. 3-8 was evident, the peak of EC cell Nos. 1 and 2 was rounded, and it was difficult to observe the peak. The shape of the peak depends on the rate of the chemical reaction when a chemical reaction occurs prior to the reversible electron transfer reaction. If the chemical reaction was slow, the voltammogram 

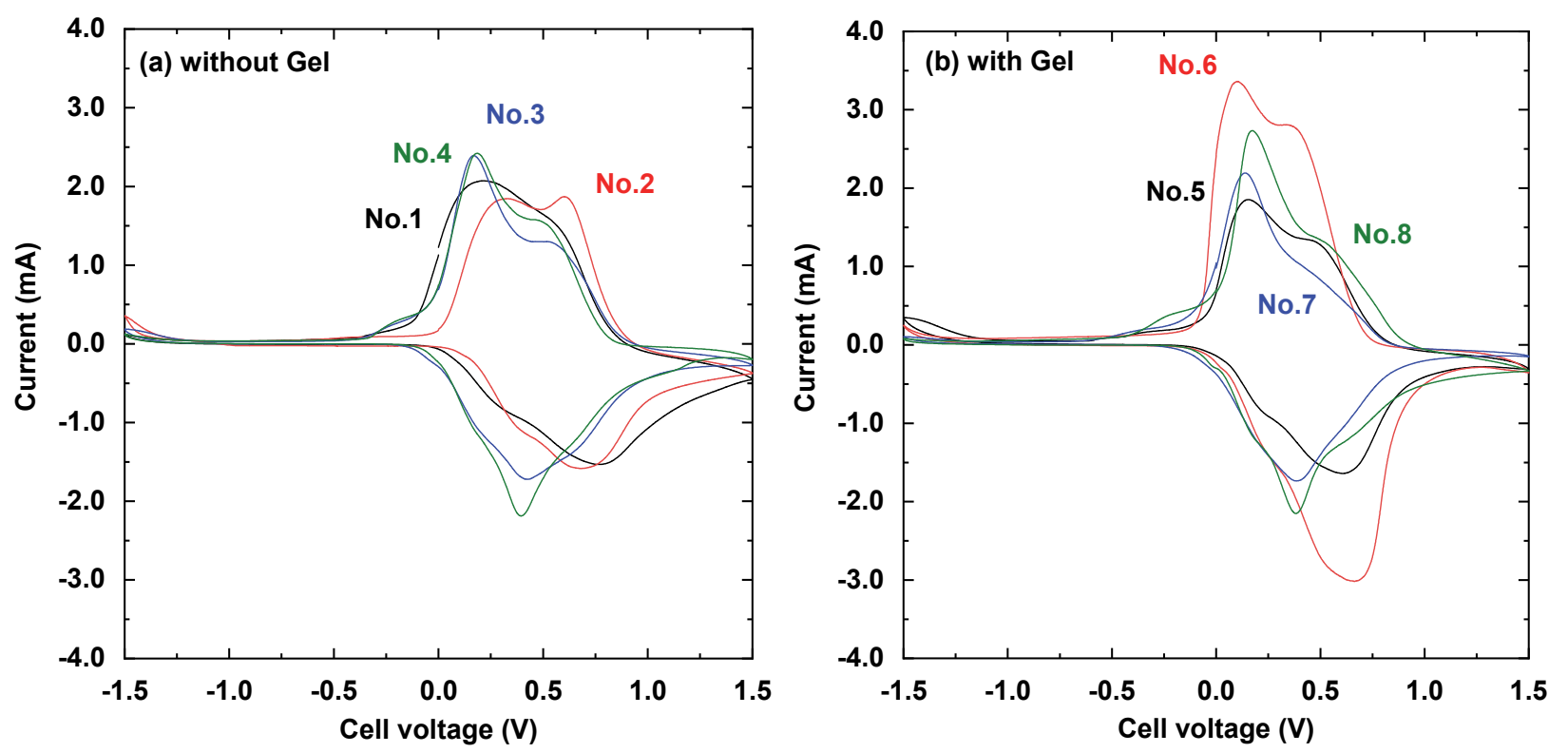

Fig. 8. Cyclic voltammograms of the EC cells (a) without polymer gel electrolyte and (b) with the gel.

did not shows a peak because the current was dominated by reaction speed. Conversely, if the chemical reaction was fast, the voltammogram exhibited a pair of oxidation peak potential and reduction peak potential. EC cell Nos. 1 and 2 were observed to exhibit extremely slow electrochromism when compared to other EC cells.

Given the EC cells other than Nos. 1 and 2, the peak potential exhibited values of $0.326-0.559 \mathrm{~V}$ in EC cell Nos. 5 and 6, and $0.214-0.253 \mathrm{~V}$ in EC cell Nos. 3, 4, 7 and 8. In the thin EC cell of the PB film, the results confirmed that separation of the peak potential decreased irrespective of the presence or absence of gelation and electrolyte concentration. Thus, the findings revealed that the chemical reaction in the PB film became fast and stabilized due to the thin PB film, and the electron transfer became fast.

Peak currents exhibited values of $-1.74--1.53$ and 1.87-2.39 mA in EC cell Nos. 3, 5, and 7, and $3.02--2.14$ and 2.41-3.32 mA in EC cell Nos. 4, 6, and 8 . A positive peak current and negative peak current were observed in all the EC cells, thereby revealing that reversible reaction occurred. The absolute value of the positive peak current increased. As shown in the chemical equation of the EC cell, the electrons are lost at $2 \mathrm{~mol} / \mathrm{L}$ in the oxidation reaction of the Ni-PBA film at the anode while the electrons are lost at $4 \mathrm{~mol} / \mathrm{L}$ in the oxidation reaction of the PB film at the cathode. Therefore, the number of electrons contributing to the reaction in the PB film increases. Thus, a positive peak exhibiting a large absolute value indicates an oxidation reaction (coloring), and a negative peak indicates a reduction reaction (decoloring).

Additionally, the difference between the positive and negative peak currents exhibited values of 6.69 $\mathrm{mA}$ in EC cell No.3, $2.62 \mathrm{~mA}$ in EC cell No.4, 3.38 $\mathrm{mA}$ in EC cell No.5, $3.03 \mathrm{~mA}$ in EC cell No.6, 4.58 $\mathrm{mA}$ in EC cell No.7, and $5.71 \mathrm{~mA}$ in EC cell No.8. Increases in the difference between the positive and negative peak current values increased the occurrence of the oxidation reaction and reduction reaction in the wide range of the EC cell. In the thin EC cell of the PB layer, the variation in the film thickness was low, and thus the oxidation reaction and reduction reaction occurred evenly over the entire film. Evidently, the thickness of the PB film affected the speed and stability of the chemical reaction. Conversely, it was evident that the type of electrolyte mainly affected the occurrence of reversible reaction in the study.

\subsection{Driving characteristics of electrochromic cells}

Finally, we fabricated the circuit shown in Fig. 9 and conducted the experiment of driving the EC cell. In contrast to the driving circuit of the inorganic EL of the previous report, we fabricated the simply ON/OFF switching circuit [19]. The input signal was controlled via PIC Microcontroller 12F629, and the program was written such that the signal oscillated alternately from the two terminals every 

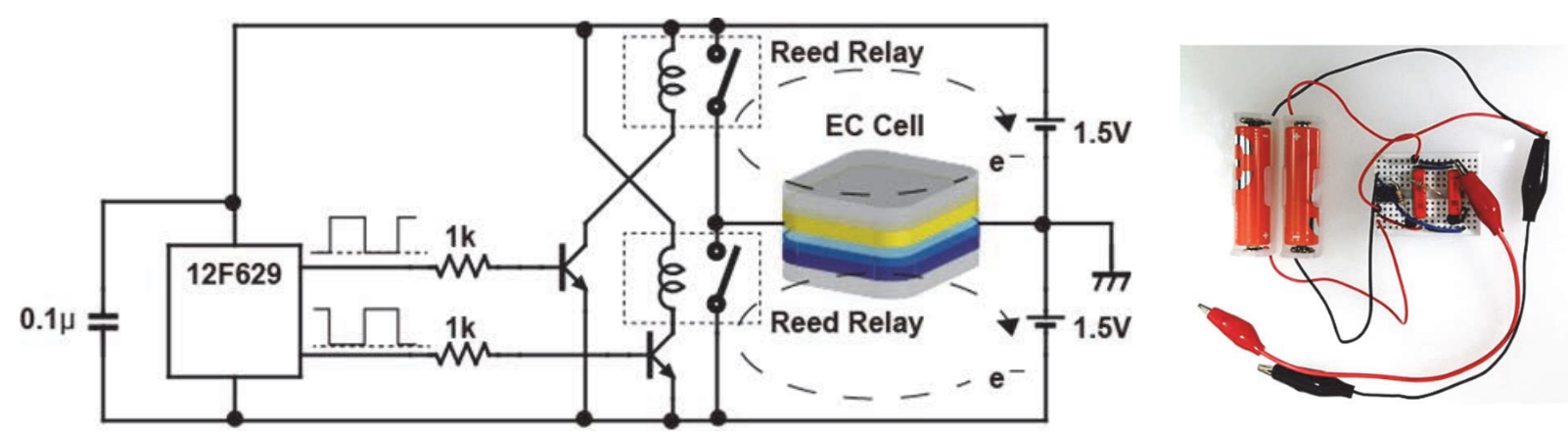

Fig. 9. Photograph and diagram of the automatic driving electronic circuit with reed relay.

$3 \mathrm{~s}$. Thereafter, a current was applied to the coil via a transistor. The coil shown in Fig. 9 is a Reed Relay that opens and closes the switch via the input signal. Switching of each Reed Relay was turned ON alternately by a signal from the microcontroller. At this instance, a driving circuit, which alternately applied $\pm 1.5 \mathrm{~V}$, was fabricated.

Measurement of responsiveness of the EC cell was performed by measuring the difference in light transmittance due to the color change that occurred with respect to the application of $\pm 1.5 \mathrm{~V}$ every $3 \mathrm{~s}$ as the voltage value of the photodiode as in the previous report [20]. Figure 10 shows the photodiode output signal with respect to the application of $\pm 1.5 \mathrm{~V}$ switched every $3 \mathrm{~s}$. The results for EC cells Nos. 1, 4, 5, and 8 are shown as a representative result. In EC cell No.1 (Fig. 10(a)), the PB film was formed at $1500 \mathrm{rpm}$. The electrolyte concentration corresponded to $0.1 \mathrm{~mol} / \mathrm{L}$. In EC cell No.4 (Fig. 10(b)), the PB film was

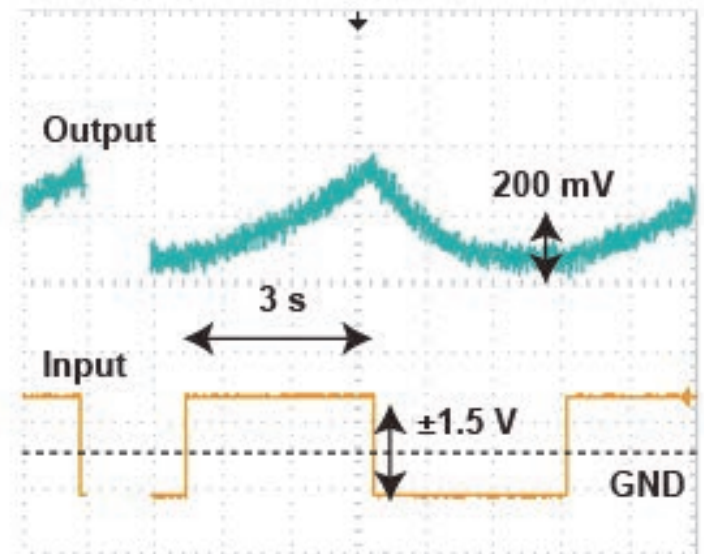

(a) No. 1

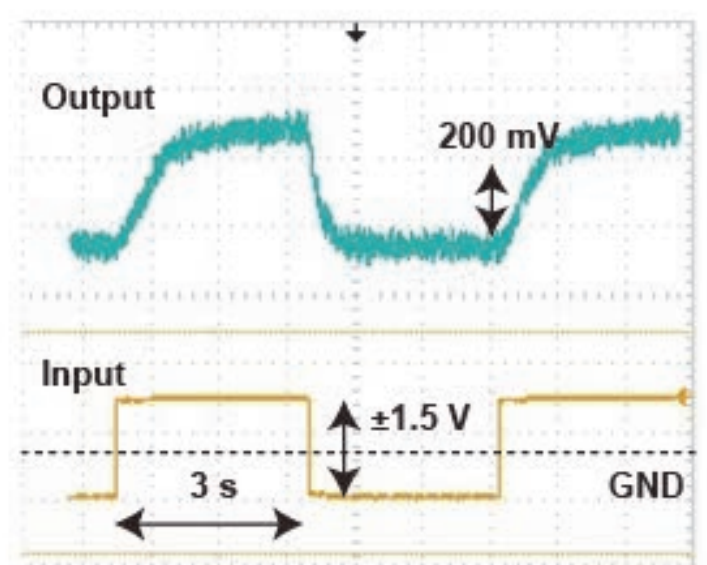

(c) No. 5

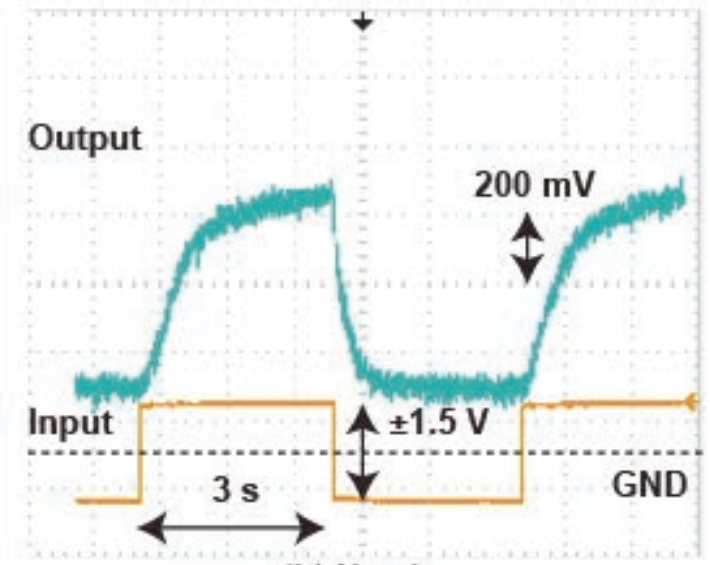

(b) No. 4
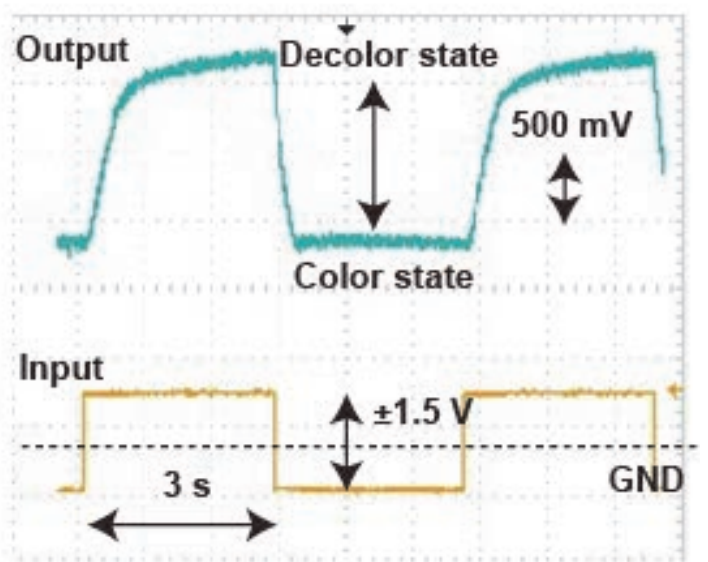

(d) No. 8

Fig. 10. Measured response times in the EC cells for (a) No. 1, (b) No. 4, (c) No. 5, and (d) No. 8. 
formed at $7500 \mathrm{rpm}$. The electrolyte concentration corresponded to $0.5 \mathrm{~mol} / \mathrm{L}$. In EC cell No.5 (Fig. 10(c)), the PB film was formed at $1500 \mathrm{rpm}$. The gel electrolyte concentration corresponded to $0.1 \mathrm{~mol} / \mathrm{L}$. In EC cell No.8 (Fig. 10(d)), the PB film was formed at $7500 \mathrm{rpm}$. The gel electrolyte concentration corresponded to 0.5 $\mathrm{mol} / \mathrm{L}$.

In all EC cells, a reversible change was observed in which the voltage value of the photodiode increased after the voltage was turned on and decreased after the voltage was turned off. However, the trend was different for each EC cell. As shown in the results of the electrical properties and chemical reactivity, the voltage value gradually increased in EC cell No. 1 (which is expected to affect the progress of the reaction slightly) and the voltage exhibited a tendency to gradually decrease after it turned OFF. Conversely, the chemical reactivity was expected to become faster in No. 4 where the thickness of the PB film was small and electrolyte concentration was high. The change sharply increased in the range of approximately $1 \mathrm{~s}$ immediately after the voltage application, and then it exhibited a two-step behavior that gradually increased while reaching the steady state until it turned OFF. The change sharply decreased in the range of approximately $1 \mathrm{~s}$ immediately after it turned OFF and exhibited a two-step behavior that gradually decreased while it reached the steady state until it turned ON. Evidently, the reactivity increased by thinning the PB film and increasing the electrolyte concentration.

Additionally, given EC cell Nos. 5 and 8 with polymer gel electrolyte, EC cell No. 5 exhibited a trend close to EC cell No. 4, and it was evident that the reactivity increased. Additionally, the change became prominent at EC cell No. 8. The evaluation device used in the study involved the observation of visual changes. In EC cell Nos. 4 and 8 , a significant difference in electrical properties was not observed although a change in appearance was significantly observed. It is inferred that changes in appearance were easier to observe because the electrolyte was immobilized by gelation.

The aforementioned measurement was conducted from EC cells No. 1 to No. 8. Table 3 summarizes the results of the coloring speed and decoloring speed. The time from when the difference between the minimum value of the voltage and maximum value after reaching the steady state changed from $10 \%$ to $90 \%$ was considered as the
Table 3. Coloring and decoloring speed of the EC cells.

\begin{tabular}{ccc} 
Cell & Coloring time & Decoloring time \\
No. & $(\mathrm{s})$ & $(\mathrm{s})$ \\
\hline 1 & \multicolumn{3}{c}{ N.D $^{\mathrm{a})}$} \\
2 & $\mathrm{~N}^{\mathrm{a}} \mathrm{D}^{\mathrm{a})}$ \\
3 & 0.52 & 2.44 \\
4 & 0.40 & 2.40 \\
\hline 5 & 0.44 & 2.32 \\
6 & 0.32 & 2.20 \\
7 & 0.20 & 2.04 \\
8 & 0.20 & 1.80 \\
\hline
\end{tabular}

a) Not determined.

measurement time.

It exhibited a tendency to increase constantly for EC cell Nos. 1 and 2, and it was impossible to measure the same because the maximum value was unknown. However, in other EC cells, the coloring time corresponded to $0.20-0.52 \mathrm{~s}$. Additionally, the decoloring time corresponded to $1.80-2.44 \mathrm{~s}$, and the coloring speed was faster. The degree was not affected by the electrolyte concentration although it was significantly affected by gelation of the electrolyte. The results indicated that the change in appearance was faster for the electrolyte with the gel. Additionally, the reason as to why coloring was faster was because 2 $\mathrm{mol} / \mathrm{L}$ of electrons are required in the oxidation reaction (coloring) while $4 \mathrm{~mol} / \mathrm{L}$ of electrons are required in the reduction reaction (decoloring). This indicated that the oxidation in which the reaction occurs with a small number of electrons proceeded faster. However, the difference was not simply doubled although it was evident that the speed ratio changed up to approximately 5-10 times based on the electrolyte concentration.

\section{Conclusion}

We prepared several types of PB-based EC cells and an automatic driving circuit. Additionally, the effect of polymer gel electrolyte on the electrical properties and driving characteristics of the EC cells was systematically investigated. This is the first study to examine the preparation of EC cells, their electrical properties, and the driving circuit. When 
the PB layer thinned, the switching speed from coloring to decoloring increased. It should be noted that the speed of coloring exceeds that of decoloring due to the electron exchange in the chemical reaction. The speed varied approximately 5-10 times based on the types of electrolytes. The thickness of PB layer significantly depended on their switching speed as opposed to the electrolyte concentration and polymer gel electrolyte. However, it was easy to observe the switch from coloring to decoloring state for the gelation of the electrolyte.

\section{Acknowledgements}

This research was partially supported by a Grantin-aid for Young Scientists B (26870611) from the Ministry of Education, Culture, Sports, Science and Technology, Japan. The authors would like to thank Mr. Hideki Miyajima at MS Scientific Co., Ltd., Tokyo, Japan for providing the date of the sedimentation velocity by LUMiSizer (LUM Japan Co., Ltd., Tokyo, Japan).

\section{References}

1. G. H. Heilmeier, IEEE T. Electron Dev., 23 (1976) 780.

2. B. J. Lechner, F. J. Marlowe, E. O. Nester, and J. Tults, Proc. IEEE, 59 (1971) 1566.

3. H. G. Slottow, IEEE T. Electron Dev, 23 (1976) 760.

4. R. Bowers and N. T. Melamed, Phys. Rev., 99 (1955) 1781.

5. E. W. Chase, R. T. Hepplewhite, D. C. Krupka, and D. Kahng, J. Appl. Phys., 40 (1969) 2512.
6. G. Gu, V. Bulović, P. E. Burrows, S. R. Forrest, and M. E. Thompson, Appl. Phys. Lett., 68 (1996) 2606.

7. C. W. Tang and S. A. VanSlyke, Appl. Phys. Lett., 51 (1987) 913.

8. M. A. Habib and S. P. Maheswari, $J$. Electrochem. Soc., 139 (1992) 2155.

9. K. C. Ho, Electrochim. Acta, 44 (1999) 3227.

10. M. Ware, J. Chem. Educ., 85 (2008) 612.

11. K. Momma and F. Izumi, J. Appl. Crystallogr., 41 (2008) 653.

12. K. Momma and F. Izumi, J. Appl. Crystallogr., 44 (2011) 1272.

13. S. Hara, H. Shiozaki, A. Omura, H. Tanaka, T. Kawamoto, M. Tokumoto, M. Yamada, A. Gotoh, M. Kurihara, and M. Sakamoto, Appl. Phys. Express, 1 (2008) 104002.

14. J. G. Jareno, J. N. Laboulais, and F. Vicente, Electrochim. Acta, 41 (1996) 835.

15. S. Skale, V. Doleček, and M. Slemnik, Corros. Sci., 49 (2007) 1045.

16. S. Agnihotry, P. Singh, A. G. Joshi, D. Singh, K. Sood, and S. Shivaprasad, Electrochim. Acta, 51 (2006) 4291.

17. K. C. Cheng, F. R. Chen, and J. J. Kai, Electrochim. Acta, 52 (2007) 3330.

18. D. J. Yang, C. Y. Hsu, C. L. Lin, P. Y. Chen, C. W. Hu, R. Vittal, and K. C. Ho, Sol. Energ. Mat. Sol. C., 99 (2012) 129.

19. S. Sato, T. Shimizu, and M. Ohuchi, J. Soc. Inf. Display, 27 (2019) 3.

20. S. Sato, T. Chikama, and M. Ohuchi, J. Photopolym. Sci. Technol., 30 (2017) 57. 